\title{
Efecto de la variación agua/cemento en el concreto
}

\author{
Génesis Guevara Fallas* \\ Carolina Hidalgo Madrigal \\ Mariannys Pizarro García \\ Iván Rodríguez Valenciano \\ Luis Diego Rojas Vega \\ Georgana Segura Guzmán
}

Fecha de recepción: 24 de mayo del 2011

Fecha de aceptación: 19 de octubre del 2011

Guevara, G; Hidalgo, C; Pizarro, M; Rodríguez, l;

Rojas, L; Segura, G. Efecto de la variación

agua/cemento en el concreto. Tecnología en Marcha.

Vol. 25, Nº 2. Abril-Junio 20 I2. Pág 80-86.

* Estudiantes de la Escuela de Ingeniería de los Materiales, Tecnológico de Costa Rica. Teléfono: 2550-22 I 3. Este trabajo corresponde al primer examen parcial del curso de Materiales I (CM-220 I), de la Escuela de Ingeniería de los Materiales, Tecnológico de Costa Rica. 


\section{Resumen}

El presente documento refleja el estudio de la relación agua-cemento $(\mathrm{A} / \mathrm{C})$ en la preparación de concreto. Para llevarlo a cabo se hicieron varias pruebas cambiando el volumen del agua con relación a la cantidad de cemento. A partir de esto se pudo concluir que el desarrollo de las pruebas permitió identificar la consistencia del concreto y, por tanto, de sus propiedades. La resistencia es afectada directamente por dicha relación y se midió mediante la prueba de compresión. Es importante determinar las propiedades del concreto con el que se está trabajando, para determinar su utilidad a largo plazo y cumplir con los estándares requeridos para tal finalidad.

\section{Palabras clave}

Prueba revenimiento, relación agua-cemento, resistencia, concreto, compresión.

\section{Abstract}

The present document reflects the study of the relation $\mathrm{A} / \mathrm{C}$ in the preparation of concrete. In order to carry it out, several tests were made changing the volume of the water relative to the amount of cement and from this it was possible to be concluded that when developing the test it allows the identification of the consistency of the concrete and therefore of its properties. The resistance is affected directly by this relation and was measured by the test of compression. It is important to determine the properties of the concrete with which one is working to determine its utility in the long term and to fulfill the standards required for such purpose.

\section{Key words}

Slump test, water-cement ratio, strength, concrete compression.

\section{Introducción}

La realización del estudio tiene una gran importancia, ya que tanto en el ámbito nacional como internacional se busca que diferentes construcciones, independientemente de su importancia, cuenten con materiales de alta calidad que cumplan los estándares establecidos por la ASTM, así como con las propiedades referentes a dureza, revenimiento, resistencia, tiempo de fraguado, etc. requeridas por las diferentes construcciones.

Es por esto que, al ser el concreto un material muy utilizado en el área de la construcción, se debe tener un claro entendimiento de él y de sus propiedades, principalmente del efecto de la relación agual cemento, pues está ligado a una gran gama de propiedades mecánicas del concreto.

No obstante, para poder iniciar el estudio de la relación agua/cemento en la fabricación de concreto, de antemano se deben conocer las propiedades y la definición de este, así como sus compuestos y las reacciones químicas que se forman para dar origen a tal material. Es por ello que a continuación se hace un resumen y se da una definición del concreto y sus respectivas reacciones.

\section{Definición del concreto y}

la relación agua-cemento

El concreto (hormigón) es un material que se obtiene de una mezcla de componentes: conglomerante (cemento), agregados (arena y piedra), agua y, de manera opcional, aditivos. La pasta, compuesta por cemento portland y agua, une los agregados, normalmente arena y grava (piedra triturada), lo cual crea una masa similar a una roca.

En esta relación agua/cemento, la importancia del agua resulta de gran magnitud, ya que ella y su relación con el cemento están altamente ligados a una gran cantidad de propiedades del material final que se obtendrá, en donde usualmente conforme más agua se adicione, aumenta la fluidez de la mezcla y, por lo tanto, su trabajabilidad y plasticidad, lo cual presenta grandes beneficios para la mano de obra; no obstante, también comienza a disminuir la resistencia debido al mayor volumen de espacios creados por el agua libre.

Así, se puede afirmar que la resistencia del concreto depende altamente de la relación por peso entre el agua y el cemento, esto se puede apreciar en el cuadro I. I 
Cuadro I. Influencia de la relación agua/cemento en la resistencia del cemento.

\begin{tabular}{|c|c|}
\hline $\mathrm{A} / \mathrm{C}$ & f'c $\left(\mathrm{kg} / \mathrm{cm}^{2}\right)$ \\
\hline 0,36 & 420 \\
\hline 0,40 & 370 \\
\hline 0,45 & 340 \\
\hline 0,50 & 295 \\
\hline 0,55 & 275 \\
\hline 0,60 & 230 \\
\hline 0,65 & 220 \\
\hline 0,70 & 185 \\
\hline 0,75 & 165 \\
\hline 0,80 & 140 \\
\hline
\end{tabular}

Asimismo, el curado es muy importante, ya que si este proceso se hace mal, se podría perder hasta el 30\% de la resistencia esperada; por eso, lo recomendable sería hacerlo por 28 días. Cabe resaltar que la resistencia que se le especifica al concreto, aproximadamente el $70 \%$, se genera en los primeros siete días. A los 14 días, la resistencia ha logrado llegar al 85\% de lo que se espera en 28 días.

En F. Smith, 1998)(I) se explica el fenómeno de la resistencia del concreto de manera similar, en donde se incorporan las reacciones químicas dadas en el proceso de formación del material, de la siguiente forma:

"El cemento portland endurece al reaccionar con agua, por las denominadas reacciones de hibridación El silicato dicálcico y el tricálcico constituyen aproximadamente un $75 \%$ en peso del cemento portland, y cuando estos componentes reaccionan con el agua durante el proceso de endurecimiento del cemento, producen como principal producto de hidratación el silicato tricálcico hidratado. Por hidratación del silicato tricálcico y el silicato dicálcico se produce también hidróxido cálcico, que es un material cristalino". Estas reacciones son:

$2 \mathrm{C}_{3} \mathrm{~S}+6 \mathrm{H}_{2} \mathrm{O} \mathrm{C}_{3} \mathrm{~S}_{2} \cdot 3 \mathrm{H}_{2} \mathrm{O}+3 \mathrm{Ca}(\mathrm{OH})_{2}$

$2 \mathrm{C}_{2} \mathrm{~S}+4 \mathrm{H}_{2} \mathrm{O} \mathrm{C}_{3} \mathrm{~S}_{2} \cdot 3 \mathrm{H}_{2} \mathrm{O}+3 \mathrm{Ca}(\mathrm{OH})_{2}$

En donde el silicato tricálcico $C_{3} S_{2} S$ endurece rápidamente y es el principal responsable de la temprana resistencia del cemento portland, y así sucesivamente con los demás productos. Por lo tanto, de las reacciones anteriores se puede aclarar que, según la extensión en que se completan las reacciones de hidratación, así se determina la resistencia y durabilidad del hormigón.
Por otro lado, un factor adicional que incide en la resistencia en los productos sólidos de la pasta de cemento hidratada es la existencia de las fuerzas de atracción de van der Waals.

Judith García (2) explica que "en efecto, la adhesión entre dos superficies sólidas se atribuye a estas fuerzas físicas, siendo el grado de la acción adhesiva función de la extensión y naturaleza de las superficies involucradas". Entonces, sabiendo que en los materiales sólidos existe una relación inversa entre la porosidad y la resistencia, esta última reside en la parte sólida de un material, por lo que lógicamente los huecos son perjudiciales para ella.

Para un volumen dado de cemento, la pasta con el mayor contenido de agua tendrá el mayor volumen total de espacio disponible, pero después de la hidratación completa, todas las pastas contendrán la misma cantidad de productos sólidos resultantes de tal proceso, por lo que la pasta con el mayor espacio total termina en un correspondiente mayor volumen de huecos capilares.

Estos espacios huecos se atan entre sí, creando la llamada porosidad. Frecuentemente, los poros crean unas quebraduras finísimas dentro del concreto, debilitándolo. Los poros están entretejidos e interconectados, permitiendo así el pasaje lento del agua a través del concreto. Cuanto más denso el concreto, más apretados los poros y menos agua puede pasar a través de ellos.

La estructura de la porosidad en el concreto influye fuertemente en su actuar. La porosidad determina las proporciones a las que las especies agresivas pueden entrar en la masa y causar destrucción.

Los índices de la intrusión se relacionan con la permeabilidad del concreto; de manera más general, la permeabilidad depende de la forma en que la porosidad total está distribuida. Esta, a su vez, se relaciona con la reacción original del cemento, las mezclas minerales y las partículas de agregados, la relación de agua-sólidos y las condiciones de curado, entre otras.

En términos de polaridad, Orozco (2004)(3) define la relación de esta con la resistencia que presentará el concreto, en donde "se afirma que la porosidad tiene efectos muy notables en la resistencia a compresión y la durabilidad del concreto. En ambas características se nota una relación inversa fundamentalmente con la porosidad. En donde la 
resistencia a compresión depende de la relación A/C pero la porosidad colabora en parte, además, la durabilidad es fuertemente amenazada por el agua que fluye a través de los sistemas de poros, que bien puede ir pura o llevar sustancias agresivas al concreto".

Para determinar las posibles porosidades presentes en el concreto, Orozco sugiere la norma ASTM C-642-97 para averiguar la indicación de la porosidad del concreto. Además, es importante conocer lo que la normativa nacional e internacional establece en cuanto a la resistencia del concreto:

- Según AC-318S-05, la resistencia mínima del concreto no puede ser menor a $173 \mathrm{~kg} / \mathrm{cm}^{2}$.

- El Código Sísmico de Costa Rica 2002 indica que debe ser de $f^{\prime} c=210 \mathrm{~kg} / \mathrm{cm}^{2}$.

- Según la norma $\mathrm{ACl} 2$ I l.I del Instituto Americano del Concreto, existe un método formal para el diseño de las mezclas de concreto.

En caso de que exista una duda, se pueden extraer núcleos del concreto, los cuales deben de ser de al menos el $85 \%$ del f'c y ninguno puede ser menor al $75 \%$.

Por otro lado, la consistencia o fluidez del concreto depende de la cantidad de agua de mezclado. El cuadro 2 muestra la consistencia numérica que se utiliza en la prueba de revenimiento, como la que se realizó para preparar el presente informe.

\begin{tabular}{|c|c|c|}
\hline Consistencia & Revenimiento & Tolerancia \\
\hline Seca & De 0 a $2 \mathrm{~cm}$ & $0 \mathrm{~cm}$ \\
\hline Plástica & De 3 a $5 \mathrm{~cm}$ & $\pm 1 \mathrm{~cm}$ \\
\hline Blanda & De 6 a $9 \mathrm{~cm}$ & $\pm 1 \mathrm{~cm}$ \\
\hline Fluida & De 10 a $15 \mathrm{~cm}$ & $\pm 2 \mathrm{~cm}$ \\
\hline Líquida & Mayor de $15 \mathrm{~cm}$ & $0 \mathrm{~cm}$ \\
\hline
\end{tabular}

Cuadro 2. La muestra obtenida en el grupo presenta una consistencia fluida.

\section{Materiales y métodos}

Para realizar las mezclas del concreto y las muestras, se emplearon como materiales cemento, arena y piedra, además de agua el componente de variación. Se realizaron dos pruebas, las cuales se describirán a continuación.

La prueba de revenimiento (cono de Abrams) consiste en tomar muestras de la mezcla de cemento que se va a analizar, debe ser aproximadamente dentro de los primeros cinco minutos después de preparar la mezcla. Se emplea un cono que se debe humedecer con agua y se sitúa en una superficie plana, lisa, húmeda y no absorbente. El cono es fabricado de acero, con un espesor mínimo de 1,5 $\mathrm{mm}$ y un recubrimiento de zinc, cuyas dimensiones son: diámetro superior $100 \mathrm{~mm}$, diámetro de la base $200 \mathrm{~mm}$ y altura $300 \mathrm{~mm}$.

Se llena el cono 1/3 del volumen, se golpea uniformemente 25 veces su interior con una barra de acero de punta hemisférica, y se golpean los lados con un mazo para distribuir la mezcla en el cono. Nuevamente este se llena 2/3 de su volumen (la mitad) y se penetra con la barra pero sin tocar la primera capa; por último, se llena el cono totalmente y se golpea sin afectar la capa anterior. Se remueve el exceso del concreto del tope del cono con una barra de acero, de manera que este quede perfectamente lleno y nivelado.

Además, se limpia el exceso de la base del molde, se retira lentamente el molde, se coloca invertido al lado de la mezcla y la barra de acero se posa sobre él. Se toma como referencia la barra de acero y se mide inferiormente la altura del cono que se formó; si existe una caída lateral o inclinación del concreto, el ensayo se descarta. La altura obtenida es la medida del revenimiento del concreto.

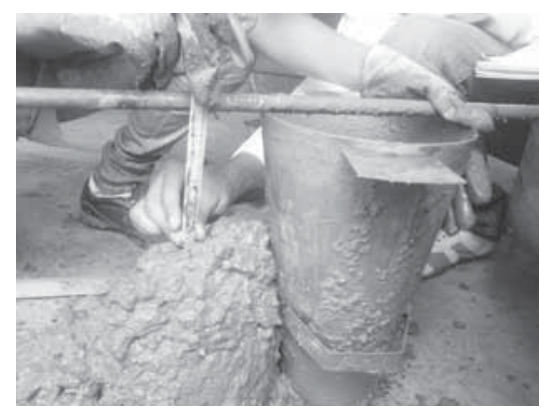

La segunda prueba por desarrollarse es la de compresión, consiste en medir la fractura de probetas cilíndricas de concreto en una máquina de ensayos de compresión. Se calcula a partir de la carga de la ruptura entre el área de la sección que recibe la carga y se mide generalmente en librafuerza pulgada cuadrada (psi) y megapascales (MPa).

Para elaborar la probeta, el cilindro debe tener las siguientes dimensiones: tamaño de $6 \times 12$ pulgadas 
Cuadro 3. Datos de los componentes utilizados para el concreto creado por cada grupo y resultados de las pruebas realizadas posteriormente, en el caso del revenimiento inmediatamente después y en el caso de la resistencia una semana después.

\begin{tabular}{|c|c|c|c|c|c|c|c|}
\hline $\begin{array}{c}N^{\circ} \text { de } \\
\text { Grupo }\end{array}$ & Relación A/C & Cemento $(\mathrm{Kg})$ & $\begin{array}{c}\text { Arena } \\
(\mathrm{Kg})\end{array}$ & $\begin{array}{c}\text { Piedra } \\
(\mathrm{Kg})\end{array}$ & $\begin{array}{c}\text { Agua } \\
(\text { Litros })\end{array}$ & Revenimiento $(\mathrm{cm})$ & $\begin{array}{c}\text { Resistencia } \\
\left(\mathrm{Kg} / \mathbf{~ m}^{2}\right)\end{array}$ \\
\hline 1 & 0.55 & 5 & 10 & 15 & 2.75 & 0 & 153.8 \\
\hline 2 & 0.60 & 5 & 10 & 15 & 3.00 & 2 & 149.3 \\
\hline 3 & 0.65 & 5 & 10 & 15 & 3.25 & 2.5 & 136.9 \\
\hline 4 & 0.70 & 5 & 10 & 15 & 3.50 & 4.5 & 122.4 \\
\hline 5 & 0.75 & 5 & 10 & 15 & 3.75 & 13 & 104.4 \\
\hline 6 & 0.80 & 5 & 10 & 15 & 4.00 & 16 & 73.6 \\
\hline 7 & 0.85 & 5 & 10 & 15 & 4.25 & 21 & 60.5 \\
\hline
\end{tabular}

$(150 \times 300 \mathrm{~mm}) \circ 4 \times 8$ pulgadas $(100 \times 200 \mathrm{~mm})$, cuando se especifique. El diámetro del cilindro debe ser como mínimo tres veces el tamaño máximo del agregado grueso que se emplea en el concreto. Un aspecto importante de esta prueba es que se pretende tener una superficie lisa para aplicar una carga uniforme; por tanto, se usan morteros de azufre o tapas de almohadillas de neopreno. Se deben aplicar como mínimo dos horas antes o preferiblemente un día antes. Se deben considerar aspectos muy relevantes antes de hacer la prueba, como:

- Los cilindros no deben estar secos.

- Los extremos de la probeta no deben tener desviaciones, sino deben estar totalmente planos.
- Se someten a prueba dos cilindros para calcular un promedio y tener mayor precisión.

\section{Resultados}

Con base en la prueba de revenimiento y de resistencia, se obtuvieron los siguientes datos que recolectan los resultados de cada una de ellas.

De acuerdo con el cuadro anterior, se elaboraron los gráficos 1 y 2 . En el primero se puede visualizar cómo al aumentar la relación A/C aumenta el revenimiento en las muestras examinadas. Además, se presenta un cambio brusco al pasar de la muestra 4 a la muestra 5.

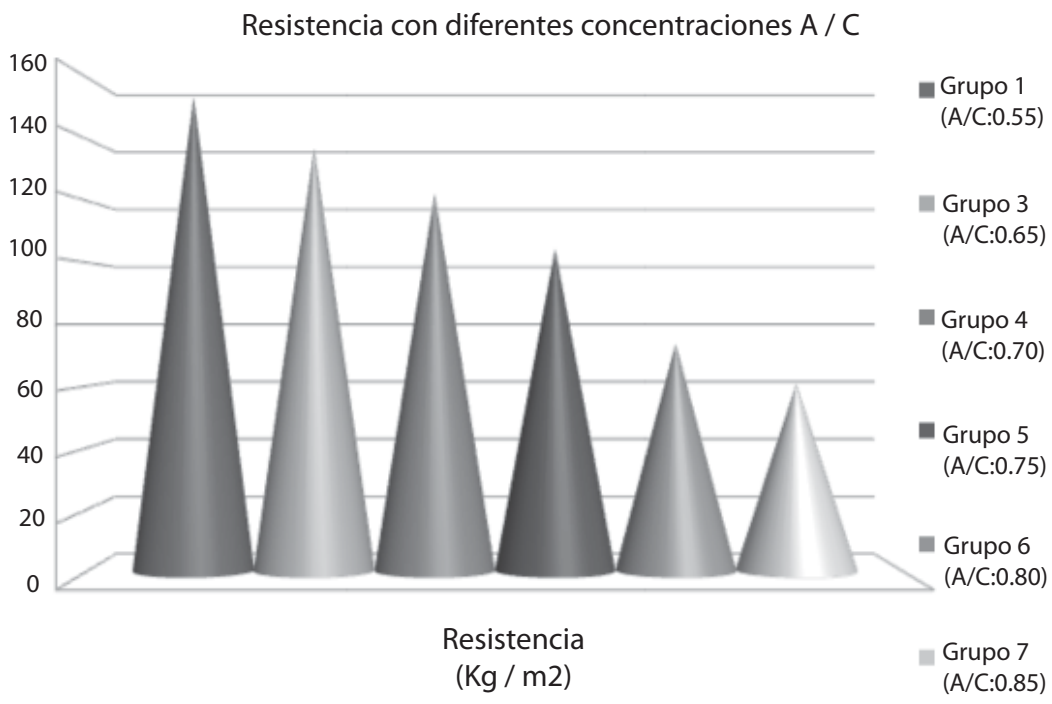




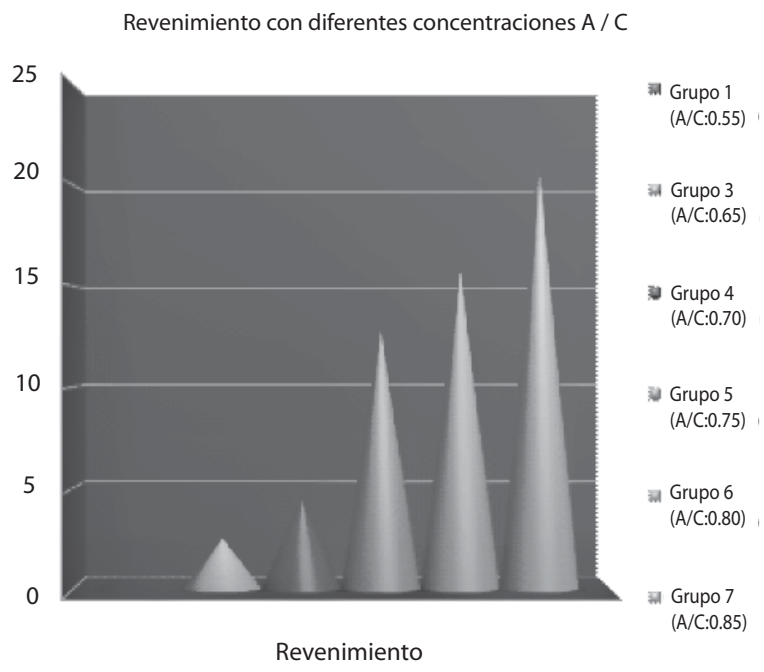

\section{Discusión y conclusiones}

Según la teoría y lo puesto en práctica sobre la creación de una mezcla de concreto, es necesario tener presentes cuatro factores muy importantes: la relación $\mathrm{A} / \mathrm{C}$, la relación cemento/agregado, la distribución granulométrica, y por supuesto la consistencia de la mezcla y resistencia del concreto.

La relación $\mathrm{A} / \mathrm{C}$, como se ve en los resultados de los distintos grupos, influye mucho en la consistencia de la mezcla, pues cuanto mayor cantidad de agua, mayor fluidez en la mezcla, mayor plasticidad y mayor trabajabilidad.

Debido a lo observado, se concluye que al alterar la relación $\mathrm{A} / \mathrm{C}$, este constituye el factor más importante que determina las propiedades del cemento, porque las reacciones de hidratación del cemento son las que determinan su resistencia; al elaborarlo, la consistencia depende de la cantidad de agua y el curado necesita de esta para mejorar las propiedades del concreto.

A partir de la prueba de revenimiento se puede determinar la consistencia del concreto y saber cómo se comportará al colocarlo. En cada caso, la medida de revenimiento cambió significativamente de una muestra a otra, variando solo la cantidad de agua y manteniendo la cantidad de los demás componentes.

De acuerdo con esta variación que se estableció, se puede interpretar que en el caso de la muestra I con la menor relación $\mathrm{A} / \mathrm{C}$ se obtuvo el menor revenimiento, lo cual significa que presenta una consistencia seca, es decir, poca cantidad de agua, y será muy difícil de trabajar.

Las muestras 2, 3 y 4 con relaciones de 0.60, 0.65 y 0.70 respectivamente presentaron una consistencia plástica, lo cual significa que fluirá sin segregarse, es decir, sin que sus componentes se separen al realizar el colocado o trabajarlo; por tanto, es muy accesible para manipular. Cabe destacar que no se presentaron muestras blandas que tengan aproximadamente un revenimiento entre 6 y $9 \mathrm{~cm}$.

La muestra 5 presenta una consistencia fluida, lo cual significa gran cantidad de agua y se manifiesta con mucha liquidez, que indica buena trabajabilidad. Las muestras 6 y 7 se consideran con consistencia líquida, ya que su revenimiento es mayor a los 15 , este concreto tiene exceso de agua y afecta todas las propiedades del concreto, pues no es trabajable y se pierde la adhesión de los componentes de la mezcla.

A partir de lo anterior se puede concluir que la prueba de revenimiento ayuda a conocer la consistencia del concreto y con ello sus propiedades. Además, la relación $\mathrm{A} / \mathrm{C}$ es el factor que afectó directamente la consistencia del concreto en las diferentes pruebas.

También la relación de cemento y agregado es de vital importancia, ya que mucha piedra o arena opacarían la cantidad de cemento a nivel micro, haciendo que este no pudiera unirse entre sí, evitando su adición. En Arqhys (sitio web de arquitectura: www.arqhys.com) se menciona que la importancia de la granulometría de los agregados radica en que de ellos dependerán las propiedades de los diferentes tipos de concretos, mayor estabilidad volumétrica y resistencia; por esto conviene que los agregados ocupen la mayor masa del hormigón, compatible con la trabajabilidad.

Por último, el factor que destaca en el concreto es su resistencia. Se determinaron pruebas de compresión a dos probetas por grupo donde se observa que laz mezclas con menor cantidad de agua tienen una resistencia a la compresión alta, ya que conforme aumenta la cantidad de agua, disminuye la resistencia a la compresión.

Esta última se halla ligada directamente a la porosidad del concreto; al tener mayor cantidad de agua en la mezcla, esta va a ocupar mucho volumen, y cuando se lleven a cabo las reacciones de hidratación, quedarán espacios vacíos en el concreto. Estos últimos se denominan poros, los cuales hacen que 
el concreto se debilite y no resista la compresión. Por tanto, en las pruebas de los primeros grupos se observa alta resistencia, porque como la mezcla es densa, no permite la formación de poros y con ello hay mayor resistencia.

A partir de esta prueba se determina la importancia de la influencia de la relación agua/cemento y la medida de resistencia de un concreto, ya que las estructuras necesitan cumplir con los códigos de sismicidad y ser resistentes a los fenómenos ambientales para perdurar mucho tiempo.

Por último, es importante recalcar que si bien se tenía planeado utilizar una relación agua/cemento de 0.5 , hubo que empezar con una de 0.55 .

La principal razón de esto es que, aunque en la teoría se diga que la relación adecuada es de 0.45, se debió elevar la relación debido a que las materias primas estaban totalmente secas, es decir, al observar la arena y la piedra, estas tienen un color blanco, con lo cual es posible afirmar que se encuentran sumamente secas.

También es importante rescatar que aunque se haya aumentado la relación A/C a 0.55 se obtiene un revenimiento de $0 \mathrm{~cm}$, y por esto se sabe que es posible aumentar esta relación de agua, pues no entra entre los 3 a $5 \mathrm{~cm}$ óptimos que se esperan en la prueba de revenimiento.

\section{Agradecimiento}

Al profesor Mauricio Araya Rodríguez, de la Escuela de Ingeniería en Construcción, por su colaboración para realizar las prácticas de laboratorio.

\section{Bibliografía}

Actualización tecnológica. Prueba de revenimiento del concreto. Extraído el 6 abril del 201 I desde http://www. basf-cc.com.mx/SiteCollectionDocuments/Boletines/ ActualTec-PruebadeRevenimientoConcreto.pdf

El concreto en la práctica ¿qué, por qué y cómo? Cip-35: Prueba de resistencia a la compresión del concreto. Extraído el 6 abril del 20I I de la web de NRMCA: http://www.nrmca.org/aboutconcrete/cips/CIP35es. pdf

Sistemas de ensayos. Extraído el 6 abril del 201 I desde http://www.sistemasdeensayo.com/productos. asp?ldFam $=2 \& \mid d$ SubFam $=22$

I - F. Smith, W. (1998). Hormigon. In W. F.Smith, \& C. F. Madriz (Ed.), Fundamentos de la Ciencia e Ingeniería de Materiales (Tercera edición ed., p. 340). Madrid: McGraw-Hill, Inc.

2- Orozco, J. О. (2004). Porosidad del concreto. Guatemala.

3- García, J. (2004). Diseño de Hormigones Dirigido a la Aplicación. Universidad Politécnica de Cataluña. 